\title{
Scintillation Light Read-Out by Low-Gain Thin Avalanche Photodiodes in Silicon Wells
}

\author{
C.P. Allier ${ }^{1}$, R.W. Hollander ${ }^{1}$, P.M. Sarro ${ }^{2}$, M. de Boer ${ }^{3}$ and C.W.E van Eijk ${ }^{1}$ \\ ${ }^{1}$ Radiation Technology Group, Interfaculty Reactor Institute, Delft University of Technology \\ Mekelweg 15, 2629 JB Delft, The Netherlands \\ ${ }^{2}$ Delft Institute of Microelectronics and Submicron Technology, Delft University of Technology, The Netherlands \\ ${ }^{3}$ MESA Research Institute, University of Twente, P.O. Box 217, 7500AE Enschede, The Netherlands
}

\begin{abstract}
We have proposed a new type of $\gamma$-ray camera, which takes advantage of micromachining technology [1]. It consists of an array of scintillator crystals encapsulated in well-type silicon sensors. The light created by the interaction of an X-ray or a gamma ray with the crystal material is confined by vertical silicon sidewalls and collected onto the avalanche photodiode at the bottom of the well. Several parameters of the photodiode need to be optimised: uniformity and efficiency of the light detection, gain, electronic noise and breakdown voltage. In order to evaluate these parameters we have processed $3 * 3$ arrays of $1.8 \mathrm{~mm}^{2}, \sim 10 \mu \mathrm{m}$ thick photodiodes using (100) wafers etched in a potassium hydroxide $(\mathrm{KOH})$ solution. Their optical response at $675 \mathrm{~nm}$ is comparable to that of a $500 \mu \mathrm{m}$ thick silicon PIN diode. The low light detection efficiency is compensated by internal amplification. Several scintillator materials have been positioned in the wells on top of the thin photodiodes, i.e. a layer of structured $\mathrm{CsI}(\mathrm{Tl})$ and single crystals of $\mathrm{CsI}(\mathrm{Tl})$ and $\mathrm{Lu}_{2} \mathrm{~S}_{3}\left(\mathrm{Ce}^{3+}\right)$. First experiments of $\gamma$-ray detection have been performed.
\end{abstract}

\section{INTRODUCTION}

3*3 arrays including $1.8 \mathrm{~mm}^{2} \mathrm{~N}^{++} \mathrm{NP}$ avalanche photodiodes, have been processed in the $\sim 10 \mu \mathrm{m}$ thick membranes that remain after thinning of $\sim 530 \mu \mathrm{m}$ thick (100) silicon wafers by means of KOH (Fig. 1). The diode characteristics have been reported in a previous work [1]. They can be summarized as follows. We measured for 5.89 $\mathrm{keV} \mathrm{X}$-rays using a calibrated charge sensitive amplifier an amplification factor of 3 close to the breakdown voltage at $67 \mathrm{~V}$. The best photodiodes have a relatively low leakage current of $0.4 \mathrm{nA}$. However all the diodes show a high junction capacitance of $\sim 50 \mathrm{pF}$ due to the thin depletion region. This results in a ENC noise of $\sim 300$ rms electrons.

In the following, the optical response of the thin test diodes is modelled taking into account the depth dependence of the gain

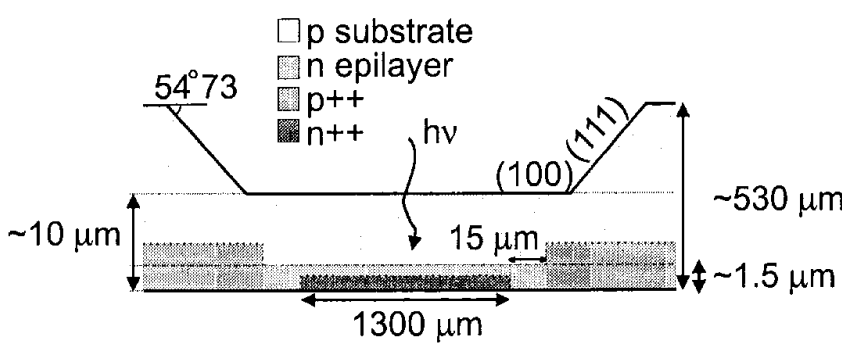

Figure 1: Schematic of the thin test photodiode (not to scale). and the presence of a silicon dead layer. Then we report the first results of scintillation light read-out obtained with the test diodes in combination with several scintillator materials, i.e. a $200 \mu \mathrm{m}$ thick film of structured $\mathrm{CsI}(\mathrm{Tl})$ and single crystals of $\mathrm{CsI}(\mathrm{Tl})$ and $\mathrm{Lu}_{2} \mathrm{~S}_{3}\left(\mathrm{Ce}^{3+}\right)$.

\section{THE OPTICAL RESPONSE}

The mean optical response, defined as the mean number of electrons per incoming photon, measured with a pulsed laser at $675 \mathrm{~nm}$ is comparable to that of $0.81 \mathrm{~cm}^{2}$ PIN photodiodes [2]. The light detection efficiency (LDE) of these PIN photodiodes is assumed to be $\sim 70 \%$. However our avalanche diodes suffer from a non-uniform optical response. When (100) wafers are etched in a $\mathrm{KOH}$ solution, gutters appear at the $(111) /(100)$ intersections which can be a few microns deep [3]. SEM membrane profiles, measured close to the tested diodes, give thicknesses of $10 \mu \mathrm{m}$ and $7.5 \mu \mathrm{m}$ for respectively the middle and the edge of the membrane. Raising the bias voltage to obtain full depletion results in an early 'breakdown' when the depletion region touches the deeper gutter where a large number of lattice defects generate a high leakage current. As a result the first few micrometers of the entrance window will remain undepleted. Consequently the light detection efficiency depends on the thickness variation of the silicon dead layer. The optical response is given by:

$$
\text { Optical response }=T \int_{0}^{W_{\text {dep }}} g(z) \lambda e^{-\lambda(W-z)} \mathrm{d} z
$$

where $z$ is the depth with the origin at the contacts of the diode, $W_{\text {dep }}$ the depletion thickness, $W$ the membrane thickness, $T$ the transmission of the $4 \mathrm{~nm}$ thick native oxide $(\sim 65 \%$ at 675 $\mathrm{nm}$ ), and $\lambda$ the light attenuation coefficient in silicon (2950 $\mathrm{cm}^{-1}$ at $675 \mathrm{~nm}$ ). The gain depth dependence $g(z)$ is derived by McIntyre [4][5]:

$$
\begin{aligned}
g(z) & =\frac{e^{-\int_{z}^{W_{\mathrm{dep}}}(\alpha-\beta) \mathrm{d} z^{\prime}}}{1-\int_{0}^{W_{\mathrm{dep}}} \alpha e^{-\int_{z^{\prime}}^{W_{\mathrm{dep}}}(\alpha-\beta) \mathrm{d} z^{\prime \prime}} \mathrm{d} z^{\prime}} \\
\alpha(z) & =2300 \cdot e^{-6.78\left(\frac{2 \cdot 10^{5}}{E(z)}-1\right)} \\
\beta(z) & =13 . e^{-13.2\left(\frac{2 \cdot 10^{5}}{E(z)}-1\right)}
\end{aligned}
$$

where $\alpha$ and $\beta$ are the ionization coefficients of electrons and holes respectively and $E$ the electric field. From a MEDICI simulation of the electric field profile, the wafer resistivity must be $4 \Omega . \mathrm{cm}$ to obtain a maximal gain calculated from Eqs. (2) of 2.9 at $67 \mathrm{~V}$ (Fig. 2). This is in agreement with 
wafer specifications. This results in a maximum of the electric field strength at $z=1.8 \mu \mathrm{m}$ of $2.710^{5} \mathrm{~V} / \mathrm{cm}$ and a depletion layer of $\sim 6 \mu \mathrm{m}$. Solving Eq. (1) for an optical response in the middle of the diode of 0.6 ( $85 \%$ compared to the PIN photodiodes), gives a membrane thickness of $\sim 9 \mu \mathrm{m}$. This is in good agreement with the SEM membrane profiles. The corresponding dead layer of $\sim 3 \mu \mathrm{m}$ results in a transmission of $40 \%$ at $675 \mathrm{~nm}$ wavelength. The model is supported by the good agreement between calculation and measurement for both gain (Fig. 2) and optical response. The gain at $675 \mathrm{~nm}$ differs from what is measured with X-rays, directly absorbed in the photodiodes. The light that is not absorbed in the dead layer may be absorbed somewhere in the high field region and experiences a mean amplification lower than the maximum gain that is measured with low-energy X-rays (Fig. 2).

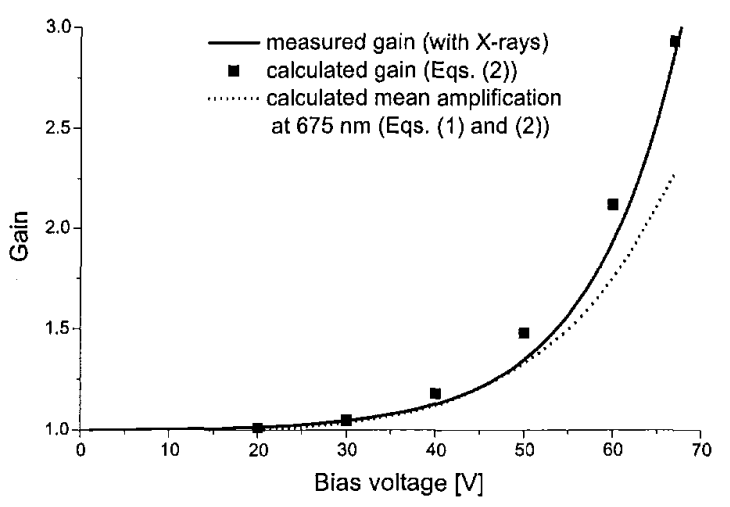

Figure 2: Comparison between the calculated and measured gain as functions of the bias voltage.

\section{SCINTILlATION Light MEASUREMENTS}

\section{A. Structured CsI (Tl)}

A $200 \mu \mathrm{m}$ thick film of structured $\mathrm{CsI}(\mathrm{Tl})$ has been deposited using a vapor deposition technique on top of thin photodiodes (Fig. 3). The attenuation length of light in silicon at $565 \mathrm{~nm}$ wavelength, the emission of $\mathrm{CsI}(\mathrm{Tl})$, is $1.5 \mu \mathrm{m}$. A

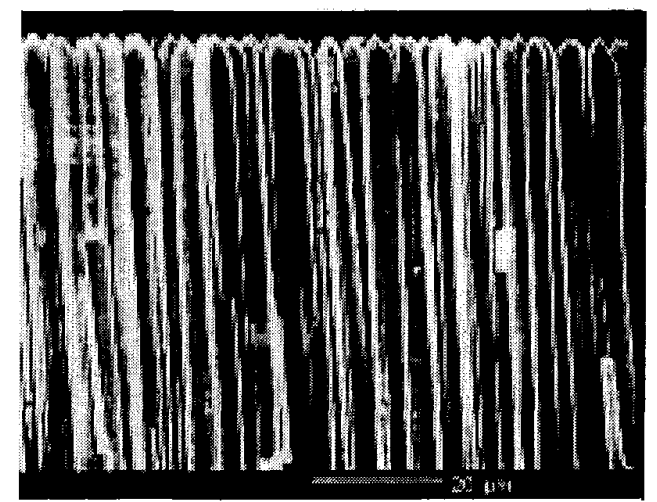

Figure 3: SEM photo of the structured CsI(Tl) (detail). By courtesy of TRIXELL. first attempt to read out scintillation light has been performed using a $5.8 \mathrm{MeV}{ }^{244} \mathrm{Cm}$ alpha source. The source was set 1.8 $\mathrm{cm}$ away from the detector in air. Each alpha deposits $\sim 4$ $\mathrm{MeV}$ in the scintillator. Assuming a light yield of $\sim 30000$ $\mathrm{ph} / \mathrm{MeV}$ [6] each event generates $\sim 120000$ photons. From the peak position in the spectrum, we calculated the number of collected electrons to be 10600 . The effective gain at $565 \mathrm{~nm}$ is similar to the maximal gain measured with X-rays, around 2 at $60 \mathrm{~V}$ bias voltage (Fig. 2). This results in a very low LDE of $4.4 \%$. One must realise that there is no reflective layer on top of the scintillator. Moreover the alpha's are absorbed in the top layer of the crystal while the surface quality and thus the light transport are rather poor. However the low LDE is mainly caused by a silicon dead layer of $\sim 3 \mu \mathrm{m}$ with a transmission of only $\sim 14 \%$. The energy resolution of $27 \%$ FWHM results from the electronic noise, the gain $g$ fluctuations, the statistical spread in the generation of photoelectrons, the spread associated with the photodetector quantum efficiency $\epsilon$, the light collection spread $R_{w}$, the non-uniform optical response contribution to the spread $R_{n u}$ and the scintillator resolution $R_{s}$ [7]:

$$
\begin{aligned}
\frac{\Delta E}{E} & =2.35 \sqrt{\left(\frac{N_{e}}{N g}\right)^{2}+\frac{(J-1)}{N}+\frac{1}{N}+\frac{1-\epsilon}{N}+R^{2}} \\
R^{2} & =R_{w}^{2}+R_{n u}^{2}+R_{s}^{2}
\end{aligned}
$$

where $N_{e}$ is the electronic noise expressed in rms electrons, $N$ the mean number of primary electrons and $J$ the excess noise factor. The detector noise spread (first two terms) is about $3 \%$ (ENC noise of $270 \mathrm{rms}$ electrons, $J \sim 1.7$ ). The statistical spread (third and fourth terms) is equal to $1.9 \%$ ( $\epsilon \sim 12 \%)$. A raytracing simulation of the light collection in a rectangular $1300 * 1300 * 200 \mu \mathrm{m}^{3}$ well sensor for interaction points $10 \mu \mathrm{m}$ deep from the surface indicates for $R_{w}$ a value around $4 \%$. The non-uniform optical response spread is around $10 \%$ (at $675 \mathrm{~nm}$ ). This is smoothed by the point-spread function wich is a $100 \mu \mathrm{m}$ FWHM Lorentzian [8]. This results in a $R_{n u}$ of $\sim 6.5 \%$. Neglecting the scintillator resolution, Eq. (3) gives for the energy resolution a value around $20 \%$ FWHM. This is rather in agreement with the measurement while the spread of the surface light losses is not taken into account in the equation. A pulse height spectrum of $356 \mathrm{keV} \gamma$-rays from a ${ }^{133} \mathrm{Ba}$ source has been measured with a shaping time of $6 \mu \mathrm{s}$ at a bias voltage of $65 \mathrm{~V}$. A $2 \mathrm{~mm}$ thick aluminium filter was used to stop low energy X-rays. The most efficient reflective top layer has been obtained by filling up the well with magnesium oxide $(\mathrm{MgO})$ powder and putting four layers of Teflon tape on top. The number of collected electrons for full energy events is $3000\left(8.5 \mathrm{e}^{-} / \mathrm{keV}\right)$. Assuming a light yield of $0.9 * 60000=54000 \mathrm{ph} / \mathrm{MeV}$ [8] each event generates $54000 * 0.356 \approx 19200$ photons. The factor 0.9 accounts for the lower yield at $6 \mu$ s shaping time due to a long decay component [9]. The detector efficiency is expressed by the factor $C . T . T_{d} . g$, where $C$ is the mean light collection, $T$ the transmission of the native oxide, $T_{d}$ the transmission of the silicon dead layer and $g$ the effective detector gain. The detector efficiency is equal to $3000 / 19200 \approx 15.5 \%$. The 
effective gain is about 2.5 at $65 \mathrm{~V}$ bias voltage. This results in a low LDE of $6.2 \%$, slightly higher than what was measured with alpha's. The difference is due to the top reflective layers that were omitted in the first measurement. With a structured CsI(TI) crystal on top of the native oxide the transmission reaches $84 \%$. The light transport in the crystal is around $50 \%$ [8]. This results in a transmission $T_{d}$ of $\sim 15 \%$ corresponding to a dead layer thickness of $2.9 \mu \mathrm{m}$. This number is given only as indication since it depends strongly on the CsI(Tl) light yield and light transport assumptions. This is however in good agreement with a dead layer of $\sim 3 \mu \mathrm{m}$ estimated for another diode from a measurements with $675 \mathrm{~nm}$ light.

\section{B. Single Crystal CsI(Tl)}

A $\sim 1^{*} 1^{*} 0.8 \mathrm{~mm}^{3} \mathrm{CsI}(\mathrm{Tl})$ single crystal has been placed in a well and coupled with viscosil $(60000 \mathrm{cst})$ to the thin photodiode. The crystal was covered with $\mathrm{MgO}$ white powder plus six layers of Teflon tape. A pulse height spectrum of 356 $\mathrm{keV} \gamma$-rays from a ${ }^{133} \mathrm{Ba}$ source has been measured with a shaping time of $6 \mu \mathrm{s}$ at a bias voltage of $66 \mathrm{~V}$. The number of collected electrons is $\sim 2700\left(7.6 \mathrm{e}^{-} / \mathrm{keV}\right)$. This is lower than what is measured with structured $\mathrm{CsI}(\mathrm{Tl})$, certainly due to the difference in light yield. The same single crystal measured with a PMT XP2254B shows a light yield around $45000 \mathrm{ph} / \mathrm{MeV}$ at $6 \mu$ s shaping time. This is around $10 \%$ lower than what is usually found in the literature. The effective gain at $66 \mathrm{~V}$ bias voltage is $\sim 2.6$. The calculated LDE is $6.5 \%$, i.e. close to what was found previously. Measurements at temperatures between 5 and $25^{\circ} \mathrm{C}$ indicate for the optical response a temperature dependence of $-0.5 \% /{ }^{\circ} \mathrm{C}$. Considering for the $\mathrm{CsI}(\mathrm{Tl})$ light yield a temperature gradient between 0.3 and 0.6 $\% /{ }^{\circ} \mathrm{C}[10]$, the temperature dependence of the detector gain is about $-1 \% /{ }^{\circ} \mathrm{C}$. This is less than the $-2 \% /{ }^{\circ} \mathrm{C}$ measured with the Hamamatsu S5345 APD [2] and $-3 \% /{ }^{\circ} \mathrm{C}$ measured by Ochi [11]. At room temperature $\left(20.5^{\circ} \mathrm{C}\right)$ we measured an energy resolution of $41 \%$ FWHM at the $356 \mathrm{keV} \gamma$-rays peak of the ${ }^{133} \mathrm{Ba}$ source. At $5{ }^{\circ} \mathrm{C}$ we found a resolution of $37 \%$. The slight improvement is mainly due to the increase of the gain. Fig. 4 shows a pulse height spectrum of $662 \mathrm{keV} \gamma$-rays from

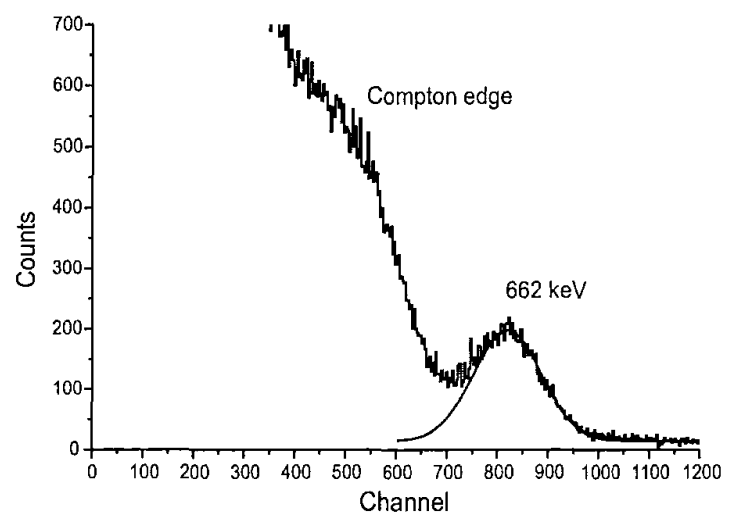

Figure 4: Pulse height spectrum of ${ }^{137} \mathrm{Cs}$ obtained with a $\sim 1 * 1 * 0.8$ $\mathrm{mm}^{3} \mathrm{CsI}(\mathrm{Tl})$ single crystal at $67 \mathrm{~V}$ bias voltage, $6 \mu$ shaping time.

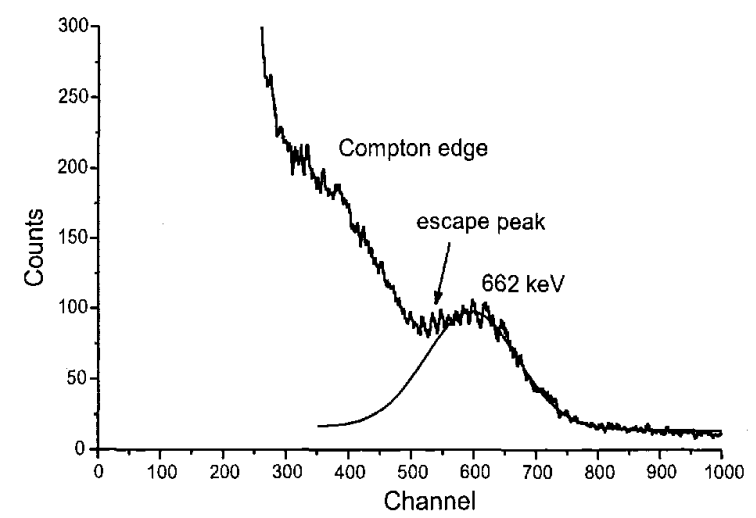

Figure 5: Pulse height spectrum of ${ }^{137} \mathrm{Cs}$ obtained with $1 * 1 * 0.5 \mathrm{~mm}^{3}$ $\mathrm{Lu}_{2} \mathrm{~S}_{3}\left(\mathrm{Ce}^{3+}\right)$ crystal at $65 \mathrm{~V}$ bias voltage, $6 \mu$ s shaping time.

a ${ }^{137} \mathrm{Cs}$ source measured at room temperature at $6 \mu$ s shaping time and $67 \mathrm{~V}$ bias voltage. The energy conversion is 7.9 $\mathrm{e}^{-/ \mathrm{keV}}$. The energy threshold at low count rates is about 250 $\mathrm{keV}$. The energy resolution measured on the high energy side of the peak is $19.1 \%$ FWHM. The detector noise contribution is $\sim 15 \%$ FWHM (ENC noise of 310 rms electrons, $J \sim 1.7$ ). One can conclude that the "light detection spread", that is $R_{w}^{2}+R_{n u}^{2}$ in Eq. (1), is lower than 5\%. This is smaller than what is found previously for structured CsI(Tl). The coupling with viscosil, the smaller area of the crystal and mainly the spread of light in the crystal reduce the non-uniform optical response contribution to the spread. The use of white powder surrounding the crystal may decrease the variance in the light collection.

\section{Single Crystal $\mathrm{Lu}_{2} \mathrm{~S}_{3}\left(\mathrm{Ce}^{3+}\right)$}

A $1^{*} 1^{*} 0.5 \mathrm{~mm}^{3} \mathrm{Lu}_{2} \mathrm{~S}_{3}\left(\mathrm{Ce}^{3+}\right)$ crystal [12] has been placed in a well. This scintillator presents a broad emission between 500 and $750 \mathrm{~nm}$. From the X-ray induced emission spectrum we have calculated the effective light attenuation length in silicon to be $1.9 \mu \mathrm{m}$. In the following we assume for this crystal a light yield of 25000 photons/MeV. The entire detector was covered with $\mathrm{MgO}$ reflective powder plus six layers of Teflon tape. We have measured pulse height spectra of 511 $\mathrm{keV}$ and $662 \mathrm{keV} \gamma$-rays from respectively ${ }^{22} \mathrm{Na}$ and ${ }^{137} \mathrm{Cs}$ sources (Fig. 5) at $6 \mu$ s shaping time and $65 \mathrm{~V}$ bias voltage (effective gain of 2.4). The $\mathrm{X}$-ray escape peaks of the $\mathrm{K} \alpha$ and $\mathrm{K} \beta \mathrm{Lu} \mathrm{X}$-rays arise $54 \mathrm{keV}$ end $62 \mathrm{keV}$ below the photopeak. The linear attenuation coefficient is $\sim 2 \mathrm{~mm}^{-1}$ for a $54 \mathrm{keV}$ $\mathrm{X}$-ray. According to Monte-Carlo GEANT simulation, we expect escape from the $\sim 0.5 \mathrm{~mm}^{3}$ crystal in $44 \%$ of the cases. The conversion is about $5.5 \mathrm{e}^{-} / \mathrm{keV}$. The energy resolution is $29 \%$ at the $662 \mathrm{keV}$ peak of ${ }^{137} \mathrm{Cs}$. Compared to $\mathrm{CsI}(\mathrm{Tl})$ measurements the light detection efficiency of $\sim 9.5 \%$ is enhanced by a factor $\sim 1.45$. According to the respective light attenuation lengths, this is in agreement with a $\sim 2.7 \mu$ m thick dead layer if we assume similar light collection efficiencies. Again this result is given only as indication, the comparison has been made for two different diodes. 
Table 1

Scintillation light read-out results.

\begin{tabular}{|c|c|c|c|c|c|c|}
\hline scintillator & radiation & $\begin{array}{l}\Delta \mathrm{E} / \mathrm{E} \\
\text { FWHM }[\%]\end{array}$ & $\begin{array}{l}\text { ENC noise } \\
\text { FWHM [\%] }\end{array}$ & $\begin{array}{l}\text { statistical noise } \\
\text { FWHM [\%] }\end{array}$ & $\begin{array}{l}\text { conversion }\left[\mathrm{e}^{-} / \mathrm{keV}\right] \\
\text { (normalised for a } \\
\text { gain of } 2.8 \text { ) }\end{array}$ & LDE [\%] \\
\hline struct. CsI(Tl) & $\begin{array}{l}5.8 \mathrm{MeV} \alpha \\
356 \mathrm{keV} \gamma\end{array}$ & $\begin{array}{l}27 \\
<50 \\
\end{array}$ & $\begin{array}{l}6 \\
21 \\
\end{array}$ & $\begin{array}{l}4 \\
9 \\
\end{array}$ & $\begin{array}{l} \\
8.5(9.4)\end{array}$ & $\begin{array}{l}4.4 \\
6.2 \\
\end{array}$ \\
\hline CsI(Tl) & $\begin{array}{l}5.8 \mathrm{MeV} \alpha \\
356 \mathrm{keV} \gamma \\
511 \mathrm{keV} \gamma \\
662 \mathrm{keV} \gamma\end{array}$ & $\begin{array}{l}17 \\
<40 \\
22 \\
19\end{array}$ & $\begin{array}{l}8 \\
27 \\
17.5 \\
14.5\end{array}$ & $\begin{array}{l}5.5 \\
10 \\
8.5 \\
7.5\end{array}$ & $\begin{array}{l}7 \\
7.6(8.2) \\
8.1(8.1) \\
7.9(7.9)\end{array}$ & $\begin{array}{l}3 \\
6.5 \\
6.4 \\
6.3\end{array}$ \\
\hline $\mathrm{Lu}_{2} \mathrm{~S}_{3}\left(\mathrm{Ce}^{3+}\right)$ & $\begin{array}{l}511 \mathrm{keV} \gamma \\
662 \mathrm{keV} \gamma\end{array}$ & $\begin{array}{l}<\overline{40} \\
29\end{array}$ & $\begin{array}{l}27 \\
19\end{array}$ & $\begin{array}{l}8 \\
6.5\end{array}$ & $\begin{array}{l}5.4(6.3) \\
5.8(6.8)\end{array}$ & $\begin{array}{l}9 \\
9.7\end{array}$ \\
\hline
\end{tabular}

\section{CONCLUSION}

The thin photodiodes combine a relatively low light detection efficiency compensated by a silicon avalanche process, which makes their optical response comparable to that of normal silicon PIN diodes for red light. The low LDE is mainly due to a dead layer of silicon between the optical entrance window and the depletion zone. From measurements with a laser at $675 \mathrm{~nm}$ we have deduced a dead layer thickness of $\sim 3 \mu \mathrm{m}$ resulting in a LDE of $\sim 27 \%$ at $675 \mathrm{~nm}$. In the read-out of $\mathrm{CsI}(\mathrm{Tl})$, considering the shorter wavelength emission $(565 \mathrm{~nm})$, the large dead layer thickness is a major drawback. From the scintillation light measurements one may deduce a dead layer thickness in the order of $2.5-3 \mu \mathrm{m}$ resulting in a transmission of only $16 \pm 3 \%$ at $565 \mathrm{~nm}$. Concerning the conversion efficiency, a mean optical response of $\sim 8.5 \mathrm{e}^{-} / \mathrm{keV}$ is obtained for $356 \mathrm{keV} \gamma$-rays with the structured CsI(Tl) (LDE of 6\%). This is smaller than what is obtained with the PIN photodiode ( $25 \mathrm{e}^{-} / \mathrm{keV}, \mathrm{LDE}$ of $55 \%$ [2]). Nevertheless an energy resolution of $19 \%$ FWHM at the $662 \mathrm{keV} \gamma$-ray peak of the ${ }^{137} \mathrm{Cs}$ source has been measured with a $\mathrm{CsI}(\mathrm{Tl})$ single crystal. In the read-out of $\mathrm{Lu}_{2} \mathrm{~S}_{3}\left(\mathrm{Ce}^{3+}\right)$, the light detection is enhanced due to the red emission. But the lower light yield makes the conversion lower than CsI(Tl), about $5.5 \mathrm{e}^{-} / \mathrm{keV}$. All measurements are summarized in Table 1. Research is now focussed on the reduction of the silicon dead layer.

\section{ACKNOWLEDGMENTS}

The authors wish to thank TRIXELL S.A. Moirans France which performs the deposition of the structured $\mathrm{CsI}(\mathrm{Tl})$ thick film. These investigations have been supported by the Netherlands Technology Foundation (STW).

\section{REFERENCES}

[1] C.P. Allier, R.W. Hollander et al., "Thin photodiodes for a scintillator-silicon well detector" Proceedings of the 1998 European Solid-State Device Research Conference, pp. 176-179, 1998.
[2] C.P. Allier, H. Valk et al., "Comparative Study of Silicon Detectors" IEEE Transactions on Nuclear Science. v $45 \mathrm{n}$ 3, pp. 576-580, 1998.

[3] B. Schmidt, J. von Borany and D. Shubert, "Application of wet chemical selective etch techniques to the fabrication of thin silicon detectors", Nucl. Instr. and Meth., A 326, pp. 21-26, 1993.

[4] R.J. McIntyre, "Multiplication Noise in Uniform Avalanche Diodes", IEEE Trans. Electron. Dev., ED-13, pp. 164-168, 1966.

[5] Y. Musienko,'Simple model of EG\&G APD", Proceedings of the Second conference on New Developments in Photodetection Beaune 99, to be published.

[6] E.V. Sysoeva, V.A. Tarasov et al., "The study of $\alpha / \gamma$ ratio for inorganic scintillation detectors", Nucl. Instr. and Meth., A 414, pp. 274-278, 1998 .

[7] C. Fiorini and F. Perotti, "Scintillation detection using a silicon drift chamber with on-chip electronics", Nucl. Instr. and Meth., A 401, pp. 104-112, 1997.

[8] Trixell, private communication.

[9] J.D Valentine, W. W. Moses et al., "Temperature dependence of $\mathrm{CsI}(\mathrm{Tl})$ gamma-ray excited scintillation characteristics", Nucl. Instr: and Meth., A 325, pp. 147-157, 1993.

[10] H. Grassmann, E. Lorenz et al., "Properties of CsI(Tl) Renaissance of an old scintillation material", Nucl. Instr. and Meth., A 228, pp. 323-326, 1985.

[11] A. Ochi, Y. Nishi, T. Tanimori, "Study of a large avalanche photodiode as a fast photon and soft X-ray detector", $\mathrm{Nucl}$. Instr. and Meth., A 378, pp. 267-274, 1996.

[12] J.C van't Spijker, P. Dorenbos et al., "Lu2S3:Ce3+, A new red luminescing scintillator", Nucl. Instr. and Meth., B 134, pp. 304-309, 1998. 